\title{
Tuning Two-Dimensional Hyperbolic Plasmons in Black Phosphorus
}

\author{
Edo van Veen, ${ }^{1,2, *}$ Andrei Nemilentsau, ${ }^{3}$ Anshuman Kumar, ${ }^{4}$ Rafael Roldán, ${ }^{5}$ Mikhail I. Katsnelson, ${ }^{2}$ \\ Tony Low, ${ }^{3}$ and Shengjun Yuan ${ }^{1,2}$ \\ ${ }^{1}$ Key Laboratory of Artificial Micro- and Nano-structures of Ministry of Education and School of Physics and \\ Technology, Wuhan University, Wuhan, China \\ ${ }^{2}$ Institute for Molecules and Materials, Radboud University, Heyendaalseweg 135, 6525AJ Nijmegen, Netherlands \\ ${ }^{3}$ Department of Electrical and Computer Engineering, University of Minnesota, Minneapolis, Minnesota 55455 , \\ USA \\ ${ }^{4}$ Physics Department, Indian Institute of Technology Bombay, Mumbai 400076, India \\ ${ }^{5}$ Materials Science Factory, Instituto de Ciencia de Materiales de Madrid (ICMM), Consejo Superior de \\ Investigaciones Cientificas (CSIC), Cantoblanco E28049, Madrid, Spain
}

(Received 11 December 2018; revised manuscript received 15 May 2019; published 8 July 2019)

\begin{abstract}
Black phosphorus presents a very anisotropic crystal structure, making it a potential candidate for hyperbolic plasmonics, characterized by a permittivity tensor where one of the principal components is metallic and the other dielectric. Here we demonstrate that atomically thin black phosphorus can be engineered to be a hyperbolic material operating in a broad range of the electromagnetic spectrum from the entire visible spectrum to ultraviolet. With the introduction of an optical gain, a new hyperbolic region emerges in the infrared. The character of this hyperbolic plasmon depends on the interplay between gain and loss along the two crystalline directions.
\end{abstract}

DOI: 10.1103/PhysRevApplied.12.014011

\section{INTRODUCTION}

Semiconducting two-dimensional (2D) crystals are excellent platforms for tuneable optoelectronics, thanks to their remarkable response to external electrical and mechanical stimuli $[1,2]$. In particular, atomically thin black phosphorus [3-6] (BP) has shown extraordinary tuneability of its optical and electronic properties by several methods [7], such as electrostatic gating [812], chemical functionalization [13], quantum confinement (number of layers) [14], external strain [15], or high pressure $[16,17]$. This allows the control of light-matter interaction in these materials, in particular the dispersion of collective polaritonic excitations [18].

Apart from being a highly tuneable optoelectronic crystal, the lattice structure of black phosphorus is very anisotropic $[1,19]$. The in-plane anisotropy implies optical birefringence, of which the extreme limit would be hyperbolicity, where the permittivity tensor has principal components of opposite sign [20-23]. Recently, in-plane hyperbolicity was implemented experimentally in the $\mathrm{GHz}$ frequency range using a metallic metasurface [24]. Moreover, in-plane hyperbolicity in natural van der Waals material $\alpha-\mathrm{MoO}_{3}$ was reported and the existence of hyperbolic surface polaritons was experimentally verified $[25,26]$. The strong anisotropy of black phosphorus suggests its

\footnotetext{
*edovanveen@gmail.com
}

potential as a natural hyperbolic material, offering new possibilities for actively manipulating polaritons in $2 \mathrm{D}$, such as directional plasmons, light emitters, superlensing effects, [23,27] etc. Hyperbolic materials possess large photonic density of states, which has been used to enhance spontaneous emission [28], nanoscale heat transfer [29], as well as for applications in broadband absorption [30] and biosensing [31]. These characteristics also appear for hyperbolic 2D materials and metasurfaces [32].

In this paper, we discuss the possibility of driving black phosphorus into the hyperbolic region via electrostatic tuning, strain, or layer number. We demonstrate that atomically thin black phosphorus can be efficiently tuned to become hyperbolic in a broad spectral range from the entire visible spectrum to the ultraviolet. In addition, the presence of a band gap in excess of the optical phonon energy lends itself as a possible $2 \mathrm{D}$ semiconductor gain medium [33,34]. With the introduction of a population inversion, we show that optical gain results in a new hyperbolic region in the infrared. Finally, we study the behavior of plasmons in both of these hyperbolic regions.

\section{OPTICAL CONDUCTIVITY AND BAND MODEL}

We describe black phosphorus by means of a $p_{z^{-}}$ orbital tight-binding model fitted to ab initio $G W$ 
methods $[35,36]$.

$$
\mathcal{H}=\sum_{i \neq j} t_{i j} c_{i}^{\dagger} c_{j}+\sum_{i \neq j} t_{p, i j} c_{i}^{\dagger} c_{j}
$$

where $c_{i}^{\dagger}\left(c_{i}\right)$ creates (annihilates) an electron at site $i$, and ten intralayer $t_{i j}$ and five interlayer $t_{p, i j}$ hopping terms are considered in the model. The band structure we obtain corresponds to an anisotropic direct band-gap semiconductor, with the gap at the $\Gamma$ point of the Brillouin zone.

The model can be straightforwardly extended to incorporate arbitrary electrostatic and strain fields. An electric field is applied by modifying the on-site potentials with $\epsilon_{i}=e \times \Delta U \times z_{i}$, where $e$ is the elementary charge, $\Delta U$ is the bias voltage (with units $\mathrm{V} / \mathrm{nm}$ ), and $z_{i}$ is the $z$ coordinate of site $i$.

On the other hand, the application of external strain leads to a variation of the interatomic bond lengths, which further modifies the hopping terms as [37]

$$
t_{i j}\left(\mathbf{r}_{i j}\right)=t_{i j}\left(\mathbf{r}_{i j}^{0}\right)\left(1-\beta_{i j} \frac{\left|\mathbf{r}_{i j}-\mathbf{r}_{i j}^{0}\right|}{\left|\mathbf{r}_{i j}^{0}\right|}\right),
$$

where $\left|\mathbf{r}_{i j}^{0}\right|$ is the distance in the equilibrium positions between two atoms $i$ and $j,\left|\mathbf{r}_{i j}\right|$ the distance in the presence of strain, and $\beta_{i j}=-d \ln t_{i j}(r) /\left.d \ln (r)\right|_{r=\left|\mathbf{r}_{i j}^{0}\right|}$ is the dimensionless local electron-phonon coupling. A microscopic estimation of $\beta_{i j}$ can be done based on the direct comparison between $a b$ initio and tight-binding calculations. Here we use $\beta \approx 4.5$ because this value was proved to give a matching between $a b$ initio and tight-binding calculations for the direct-to-indirect band-gap transition under uniaxial strain [38]. The mechanical properties of black phosphorus are highly anisotropic, with zigzag direction being about four times stiffer than armchair direction [15,39]. Therefore, we use uniaxial strain along the armchair direction for our calculations, accounted for by the strain tensor $\epsilon_{\mathrm{ac}}=\epsilon_{y y} \operatorname{diag}\left(\nu_{z}^{\mathrm{ac}}, 1,-v_{z}^{\mathrm{ac}}\right)$, where the Poisson ratios $v$ are estimated to be $v_{x}^{\mathrm{ac}} \approx 0.2$, and $\nu_{z}^{\mathrm{ac}} \approx 0.2$ [39]. We notice the importance of considering the out-of-plane Poisson ratio $v_{z}$ in our calculations, which accounts for the widening (flattening) of the lattice under compressive (tensile) strain.

The optical conductivity in the zigzag $\left(\sigma_{x x}\right)$ and armchair $\left(\sigma_{y y}\right)$ directions is given by the Kubo formula

$$
\begin{aligned}
\operatorname{Re}\left[\sigma_{\alpha \alpha}(\omega)\right]= & -\frac{g_{S}}{\Omega \omega} \int_{\mathrm{BZ}} \operatorname{Im}\left[\sum_{i, j}\left|\left\langle\mathbf{k} i\left|J_{\mathbf{k} \alpha}\right| \mathbf{k} j\right\rangle\right|^{2}\right. \\
& \left.\times \frac{f\left(E_{\mathbf{k} i}-\mu\right)-f\left(E_{\mathbf{k} j}-\mu\right)}{E_{\mathbf{k} i}-E_{\mathbf{k} j}+\omega+i \delta}\right] d^{2} \mathbf{k} .
\end{aligned}
$$

Here, $|\mathbf{k} i\rangle$ and $E_{\mathbf{k} i}$ are the eigenstates and eigenenergies for momentum $\mathbf{k}$ and orbital $i . g_{S}=2$ is the spin degeneracy, $\Omega$ is the unit cell surface, and $\delta=5 \mathrm{meV}$ is a small damping parameter. $J_{\mathbf{k} \alpha}$ is the current operator in the $\alpha$ direction:

$$
J_{\mathbf{k} \alpha}=-\frac{i e}{\hbar} \sum_{i, j} e^{i\left(\mathbf{r}_{j}-\mathbf{r}_{i}\right)_{\alpha} \cdot \mathbf{k}} t_{i j}\left(\mathbf{r}_{j}-\mathbf{r}_{i}\right)_{\alpha} c_{\mathbf{k} i}^{\dagger} c_{\mathbf{k} j} .
$$

Moreover, $f(E-\mu)$ is the Fermi-Dirac distribution with Fermi level $\mu$ :

$$
f(E-\mu)=\frac{1}{e^{(E-\mu) / k T}+1},
$$

where we set $T=300 \mathrm{~K}$.

We can replace $f(E)$ with a quasiequilibrium distribution $n_{F}(E, \Delta \mu)$ to introduce population inversion, which will produce optical gain $[40,41]$ :

$$
\begin{aligned}
n_{F}(E)= & \theta(E) f\left(E+\frac{E_{g}}{2}+\Delta \mu\right) \\
& +\theta(-E) f\left(E-\frac{E_{g}}{2}-\Delta \mu\right),
\end{aligned}
$$

where $E_{g}$ is the band gap.

Using the Kramers-Kronig relations, we can also obtain the imaginary part:

$$
\operatorname{Im}\left[\sigma_{\alpha \alpha}(\omega)\right]=-\frac{2 \omega}{\pi} \mathcal{P} \int_{0}^{\infty} \frac{\operatorname{Re}\left[\sigma_{\alpha \alpha}\left(\omega^{\prime}\right)\right]}{\omega^{\prime 2}-\omega^{2}} d \omega^{\prime} .
$$

\section{HYPERBOLIC REGIONS}

We first define the condition for hyperbolicity. We note that the real part of the dielectric permittivity is proportional to $\operatorname{Im}(\sigma)$, a consequence of current continuity. Then, a hyperbolic region appears when

$$
\operatorname{Im}\left[\sigma_{x x}(\omega)\right] \times \operatorname{Im}\left[\sigma_{y y}(\omega)\right]<0 .
$$

On the other hand, $\operatorname{Re}(\sigma)$, is directly proportional to the optical absorption of the free-standing 2D layer. For pristine bilayer BP, the optical conductivity components are plotted in Fig. 1.

The first thing we observe is that the peculiar puckered structure of black phosphorus leads to a strong linear dichroism, i.e., a large difference in optical conductivity for incident polarized light along armchair and zigzag directions [42]. For a bilayer sample, its optical absorption revealed two sharp peaks along the armchair direction, due to the two interband excitations indicated in red in the band structure. These resonantlike features, for light polarized along the armchair direction, has also been observed experimentally [43]. In contrast, light polarized along the zigzag 

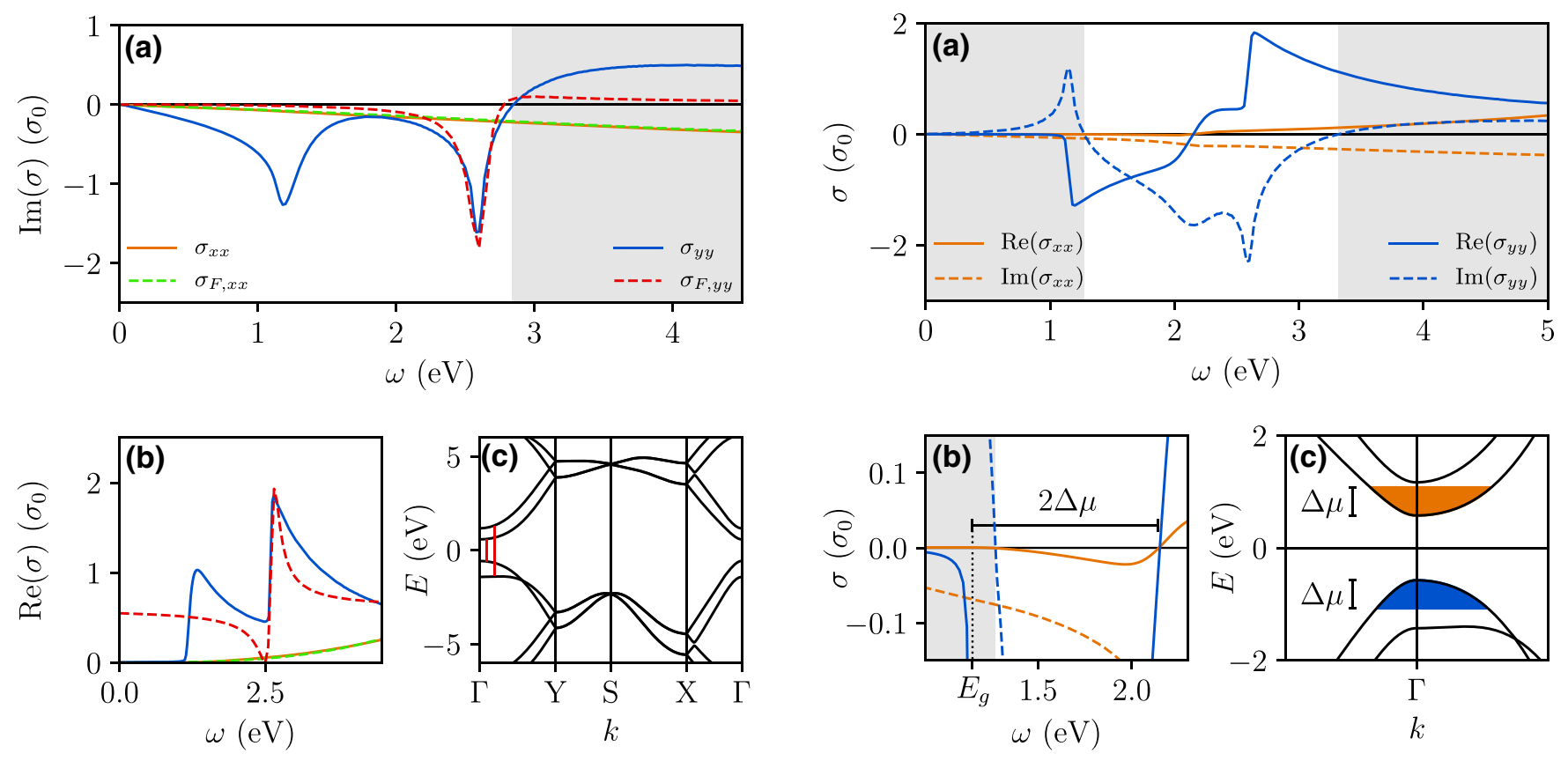

FIG. 1. (a) The imaginary part of the optical conductivity of bilayer black phosphorus in units of $\sigma_{0}=\pi e^{2} / 2 h$, showing a hyperbolic region (shaded) where $\operatorname{Im}\left(\sigma_{x x}\right) \times \operatorname{Im}\left(\sigma_{y y}\right)<0$, starting at $\omega=2.8 \mathrm{eV}$. (b) The real part of the conductivity corresponding to (a). The dashed lines show a fit of the conductivity around the second peak, using the Fano model as described in the text, with resonance width $\Gamma_{\text {res }}=0.1 \mathrm{eV}$, Fano parameter $q_{F}=1.5 \mathrm{eV}^{-1}$, and $n=3$. (c) The corresponding band structure, with optical excitations indicated in red, causing the two optical peaks at $\omega=1.2 \mathrm{eV}$ and $\omega=2.6 \mathrm{eV}$.

direction shows a featureless monotonically increasing optical absorption instead. Whereas $\operatorname{Im}\left(\sigma_{x x}\right)$ is negative throughout the spectrum, $\operatorname{Im}\left(\sigma_{y y}\right)$ goes from negative to positive around $\omega_{h}=2.8 \mathrm{eV}$, which results in a hyperbolic region starting at that frequency.

The sign change in $\operatorname{Im}\left(\sigma_{y y}\right)$ along the armchair direction is key to the appearance of the hyperbolic region as indicated in Fig. 1. This can be traced to the resonantlike feature in the optical absorption $\operatorname{Re}\left(\sigma_{y y}\right)$ at $\omega_{\text {res }}=2.6 \mathrm{eV}$. The spectral shape of $\operatorname{Re}\left(\sigma_{y y}\right)$ can be described by a Fano resonance curve

$$
\sigma_{F, y y} \sim \frac{\left(q_{F} \Gamma_{\text {res }} / 2+\omega-\omega_{\text {res }}\right)^{2}}{\left(\Gamma_{\text {res }} / 2\right)^{2}+\left(\omega-\omega_{\text {res }}\right)^{2}}
$$

and setting $\sigma_{F, x x} \sim \omega^{n}$. A fit of these curves to the region around the second peak in the optical conductivity of bilayer black phosphorus is shown by the dashed lines in Fig. 1. Its Kramers-Kronig pair, which correponds to $\operatorname{Im}\left(\sigma_{y y}\right)$, reveals a sign change after $\omega_{\text {res. }}$. Hence, we can attribute the origin of hyperbolicity to the strong and anisotropic resonantlike interband transitions.

If we introduce population inversion [Eq. (6), Fig. 2], however, the situation becomes qualitatively different: a
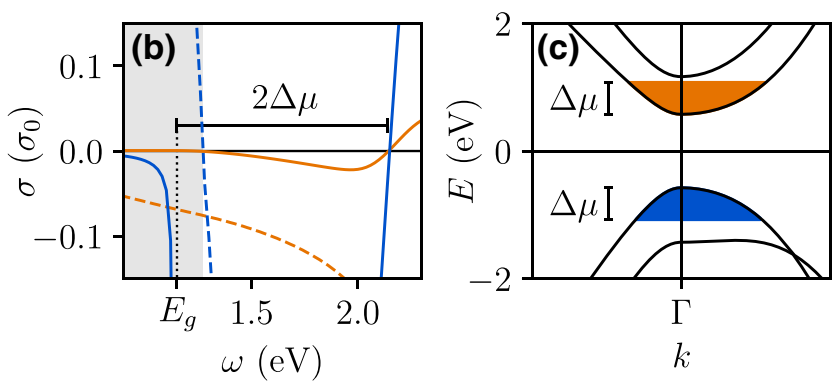

FIG. 2. (a) The optical conductivity of bilayer black phosphorus with photodoping $\Delta \mu=0.5 \mathrm{eV}$. (b) A close-up of the region where $\operatorname{Re}\left(\sigma_{y y}\right)<0$, showing a new hyperbolic region (shaded) for $\omega<1.27 \mathrm{eV}$. (c) The corresponding band structure around the $\Gamma$ point, with the population-inverted pockets shown in blue and orange.

new hyperbolic region appears in the infrared range. Here, we assume that the quasi-Fermi levels are such that $\mu_{h}=$ $\mu_{e}$, and that the electron and hole baths can be described by a common temperature [see Fig. 2(c)]. Optical pumping $[44,45]$, where electrons and holes are generated in pairs, of a charge neutral system with particle-hole symmetry would fit such a scenario. The optical gain causes $\operatorname{Re}\left(\sigma_{y y}\right)$ to become negative. The spectral window where $\operatorname{Re}\left(\sigma_{y y}\right)<0$ roughly coincides with $E_{g}<\omega<E_{g}+2 \Delta \mu$, where optical transitions between the population inverted electron and hole bands are allowed. In the region up to $\omega=1.27 \mathrm{eV}$, we find that $\operatorname{Im}\left(\sigma_{y y}\right)>0$. In the zigzag direction, the real part of the conductivity also flips sign between $E_{g}<\omega<E_{g}+2 \Delta \mu$. The imaginary part in this direction, on the other hand, remains negative throughout the entire frequency range, causing a new hyperbolic region in the infrared.

Since the origin of the hyperbolicity is related to the strong resonantlike anisotropic interband absorption between the largest conduction and valence subband indices, one expects that the spectral range of hyperbolicity can be tuned with band-structure engineering. Indeed, the onset of the hyperbolic region $\omega_{h}$ can be tuned with the number of layers, strain, bias, and doping (Fig. 3). For example, as the results show in Figs. 4(c) and 4(d), under compressive strain the band gap of bilayer black phosphorus becomes smaller. As a result, the optical peaks shift to 


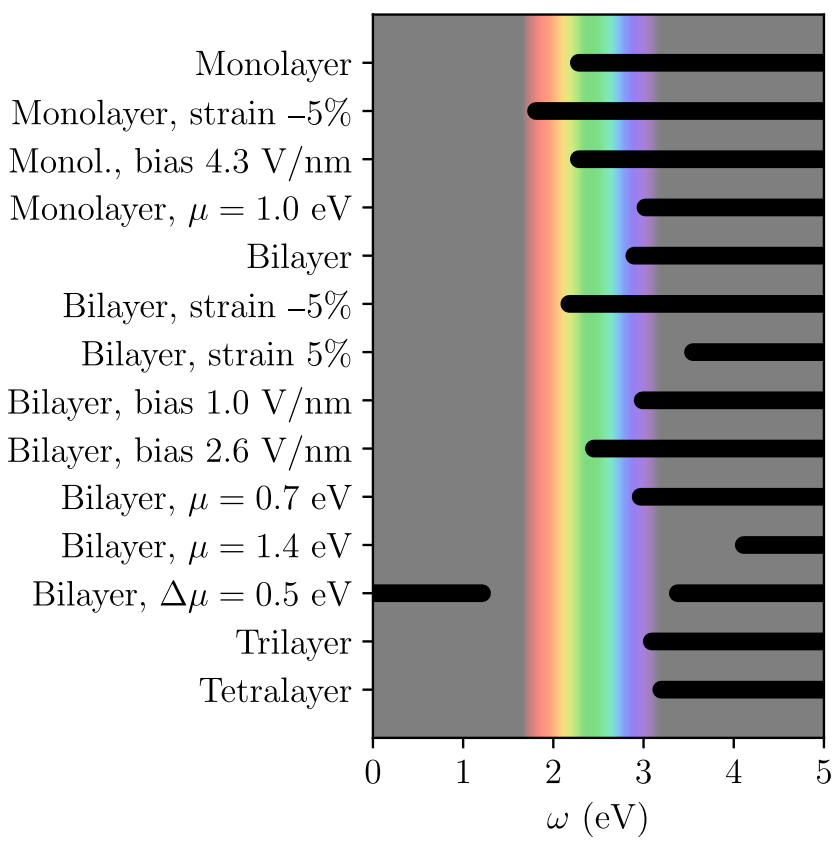

FIG. 3. The hyperbolic region (indicated in black lines) for different tuning parameters. The visual spectrum is indicated in color.

lower frequencies and $\omega_{h}$ goes down [15]. For increasing bias in the bilayer case [Figs. 4(e) and 4(f)], the hyperbolic onset frequency $\omega_{h}$ first goes up, because the bands corresponding to the excitation causing the second peak move away from one another. Then it goes down as the band gap closes and a new peak appears in between the two existing peaks, because breaking the mirror symmetry in the $z$ direction allows for new hybrid transitions [43]. Moreover, $\omega_{h}$ goes up for an increasing number of layers, because extra layers add peaks to the optical conductivity in the armchair direction, and the hyperbolic region appears after the last peak. Finally, for increased doping, $\omega_{h}$ moves further up, as the first peak becomes less prominent due to Pauli blocking.

\section{HYPERBOLIC PLASMONS}

Finally, let us consider characteristics of plasmons that can be supported by a hyperbolic material. We assume that the plasmon propagates at an angle $\chi$ with respect to the $x$ axis, where the plasmon wave vector takes the form $\mathbf{q}=q_{x} \mathbf{e}_{x}+q_{y} \mathbf{e}_{y}+i \mathbf{e}_{z} \gamma$, where $q_{x}=q_{\|} \cos \chi, q_{y}=$ $q_{\|} \sin \chi, q_{\|}=\sqrt{q_{x}^{2}+q_{y}^{2}}$. The dispersion relation for the hyperbolic plasmon takes form

$$
q_{\|}^{2}=\gamma^{2}+k_{0}^{2},
$$

where

$$
\begin{gathered}
\gamma=\frac{i k_{0}}{2 \sigma_{\mathbf{q q}}}\left[\left(\frac{2}{\eta_{0}}+\frac{\eta_{0}}{2} \sigma_{x x} \sigma_{y y}\right)\right. \\
+\sqrt{\left.\left(\frac{2}{\eta_{0}}+\frac{\eta_{0}}{2} \sigma_{x x} \sigma_{y y}\right)^{2}-4 \sigma_{\mathbf{q q}} \sigma_{\perp \perp}\right]}, \\
k_{0}^{2}=\omega^{2} \mu_{0} \varepsilon_{0}, \text { and } \\
\sigma_{\mathbf{q q}}(\chi)=\sigma_{x x} \cos ^{2} \chi+\sigma_{y y} \sin ^{2} \chi \\
\sigma_{\perp \perp}(\chi)=\sigma_{x x} \sin ^{2} \chi+\sigma_{y y} \cos ^{2} \chi .
\end{gathered}
$$

The isofrequency contours $\left[\omega\left(q_{x}, q_{y}\right)=\right.$ constant], calculated using Eq. (10), are presented in Fig. 5. We consider the cases of black phosphorus with gain $\left[\sigma_{x x}=(0.07-\right.$ i6.4) $\left.\times 10^{-2} \sigma_{0}, \sigma_{y y}=(-0.03+i 0.60) \sigma_{0}\right]$ and black phosphorus under strain $\left[\sigma_{x x}=(0.20-i 0.31) \sigma_{0}, \sigma_{y y}=(0.20+\right.$ $i 0.54) \sigma_{0}$ ]. It can be seen from Figs. 5(a) and 5(b), that only in the case of black phosphorus with gain the isofrequency contour resembles a hyperbola with the asymptotes defined as

$$
\tan \chi_{0}=\sqrt{\left|\frac{\operatorname{Im}\left(\sigma_{x x}\right)}{\operatorname{Im}\left(\sigma_{y y}\right)}\right|} .
$$

For black phosphorus under strain, the isofrequency contour resembles a figure-of-eight shape, even though the hyperbolicity condition (8) is met.

This behavior stems from the fact that the hyperbolic shape of the isofrequency contour is related to the poles of the denominator in Eq. (11), defined by zeros of $\sigma_{\mathbf{q q}}$ (i.e., when $\sigma_{\mathbf{q q}} \rightarrow 0$, then $|\gamma| \rightarrow \infty$ and $\left|q_{\|}\right| \rightarrow \infty$ ). In particular, it is straightforward to demonstrate that in the case of a purely imaginary conducitivity tensor (i.e., no losses or gain) the condition $\sigma_{\mathbf{q q}}=0$ leads to Eq. (14) for hyperbola asymptotes.

If the components of the conductivity tensor are both lossy $\left[\operatorname{Re}\left(\sigma_{x x, y y}\right)>0\right]$ or both have gain $\left[\operatorname{Re}\left(\sigma_{x x, y y}\right)<0\right]$, the module of the conductivity, $\left|\sigma_{\mathbf{q q}}\right|$, is never zero. In fact, for the hyperbola asymptote angle $\chi_{0},\left|\sigma_{\mathbf{q q}}\left(\chi_{0}\right)\right|=$ $\left|\operatorname{Re}\left(\sigma_{x x}\right)\right| \cos ^{2} \chi_{0}+\left|\operatorname{Re}\left(\sigma_{y y}\right)\right| \sin ^{2} \chi_{0}$. Thus, $\left|\sigma_{\mathbf{q q}}\left(\chi_{0}\right)\right|$ increases with the increase of $\left|\operatorname{Re}\left(\sigma_{x x, y y}\right)\right|$ both for the lossy material and the material with gain. This leads to the decrease of $q_{\|}\left(\chi_{0}\right)$ and, eventually, destroys hyperbolicity when either losses or gain are too high. For example, this is the case in black phosphorus with strain presented in Figs. 5(b) and 5(d). The high losses of the material in the hyperbolic regime lead to the hyperbola folding into a figure-of-eight-shape isofrequency contour. Moreover, the plasmons itself are very lossy in this case as is quantified by ratio $\operatorname{Re} q_{\|} / \operatorname{Im} q_{\|}$in Fig. 5(d).

The case where one of the components of the conductivity tensor is lossy $\left[\operatorname{Re}\left(\sigma_{x x}\right)>0\right]$, while the other 


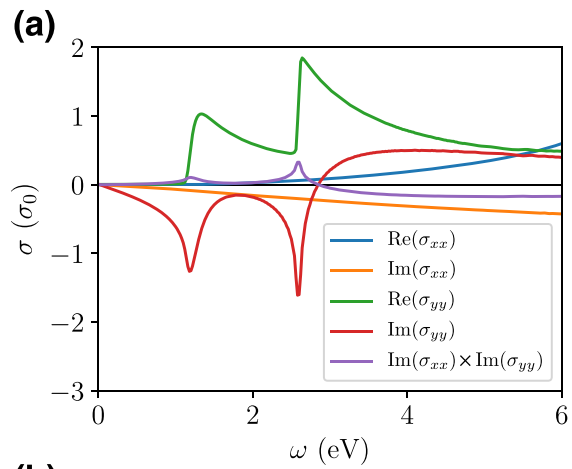

(b)

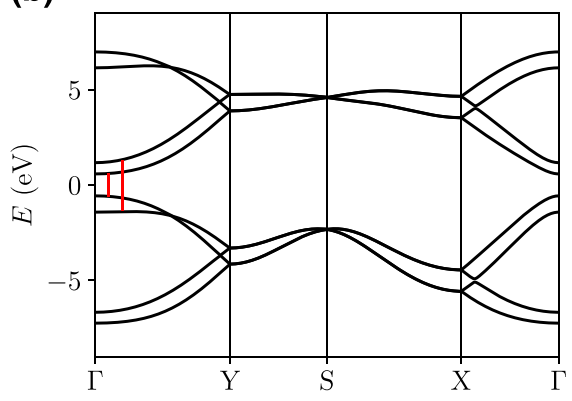

(c)

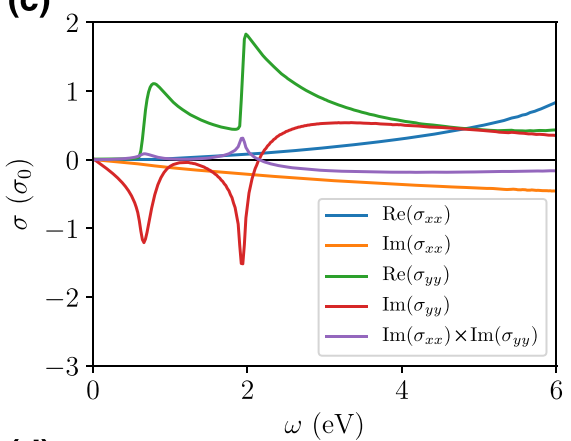

(d)

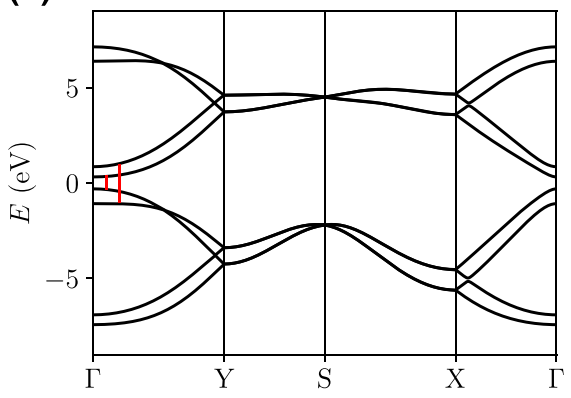

(e)

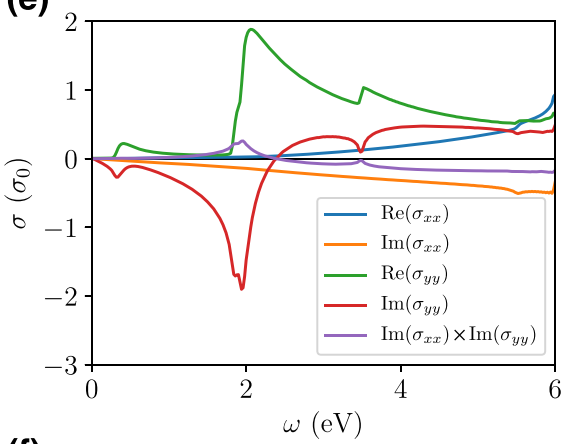

(f)

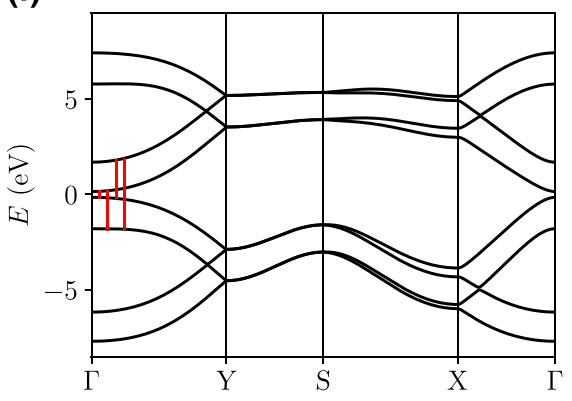

FIG. 4. The optical conductivity of bilayer black phosphorus (a) pristine; (c) with strain $\epsilon_{y y}=-5 \%$; (e) with a bias of $2.6 \mathrm{~V} / \mathrm{nm}$. The corresponding band structures are given in (b),(d), and (f), respectively, with optical excitations indicated in red.

has gain $\left[\operatorname{Re}\left(\sigma_{y y}\right)<0\right]$, requires separate consideration. In this case, $\sigma_{\mathbf{q q}}\left(\chi_{0}\right)=\left|\operatorname{Re}\left(\sigma_{x x}\right)\right| \cos ^{2} \chi_{0}-\left|\operatorname{Re}\left(\sigma_{y y}\right)\right| \sin ^{2} \chi_{0}$. When both the losses and the gain are small, then $\left|\sigma_{\mathbf{q q}}\left(\chi_{0}\right)\right|$ is small as well, which allows for the isofrequency contour

(a) Gain, $\omega=1.08 \mathrm{eV}$

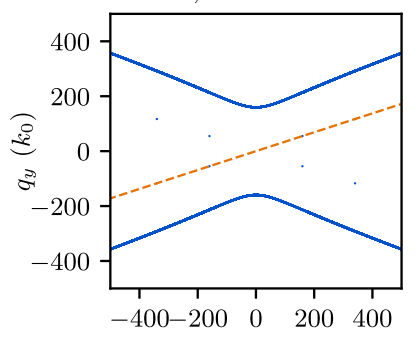

(b) Strain, $\omega=3.2 \mathrm{eV}$
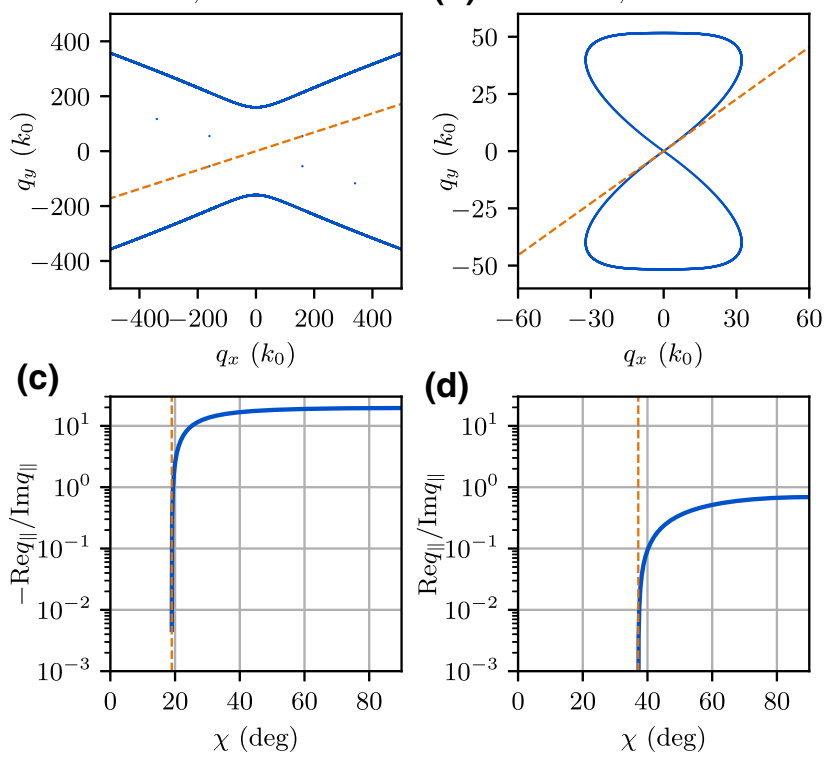

(d)

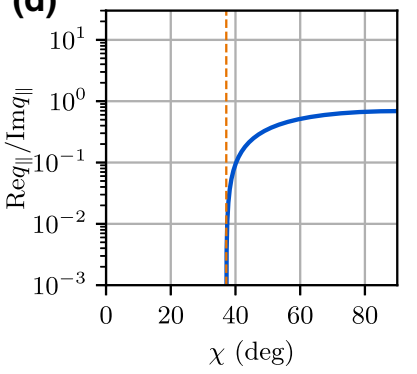

FIG. 5. Isofrequency contours and figures of merit for hyperbolic materials with gain $(\Delta \mu=0.5 \mathrm{eV})$ and $\operatorname{strain}\left(\epsilon_{y y}=-5 \%\right)$, calculated using Eq. (10). The hyperbola asymptotes (orange dashed lines) are defined using Eq. (14). to preserve the hyperbolic shape, as is the case for black phosphorus with gain presented in Figs. 5(a) and 5(c). This is, however, a rather trivial case, which can be observed in pure lossy materials when the losses are small [23]. A nontrivial property of a material with gain is that $\sigma_{\mathbf{q q}}=0$, when $\tan \chi_{0}=\sqrt{\left|\operatorname{Re}\left(\sigma_{x x}\right)\right| /\left|\operatorname{Re}\left(\sigma_{y y}\right)\right|}$. That condition, together with Eq. (14), indicates that the isofrequency contour preserves its hyperbolic shape for arbitrary large losses and gain, as long as the following holds true:

$$
\sigma_{x x}=-\sigma_{y y} \tan \chi_{0} .
$$

This equation is indeed beneficial for the case of the hyperbolic plasmons when one of the components of the conductivity tensor has gain, while the other one is lossy, by considering isofrequency contours for the materials with different values of gain. To illustrate this, we choose black phosphorus under strain, and assume that a gain is added to the $y$ component of the black phosphorus conductivity, with the gain values $\left[\operatorname{Re}\left(\sigma_{y y}\right)\right]$ indicated in the legend of Fig. 6. Due to high losses, the isofrequency contour of black phosphorus under strain does not resemble a hyperbola. Countering the high losses with high gain is not beneficial for restoring the hyperbolic plasmons [see Fig. 6(a)] as this leads to a high magnitude of $\sigma_{\mathbf{q q}}$. However, we can recover the hyperbolic mode by matching $\sigma_{x x}$ to $\sigma_{y y}$ using Eq. (15), as can be see from Fig. 6(b) $\left[\operatorname{Re}\left(\sigma_{y y}\right)=-20 \mu \mathrm{S}\right]$. A further decrease of gain breaks the resonance condition (15) and leads to distortion of the hyperbola $[-10 \mu \mathrm{S}$ and $-1 \mu \mathrm{S}$ in Fig. 6(b)]. 
(a)

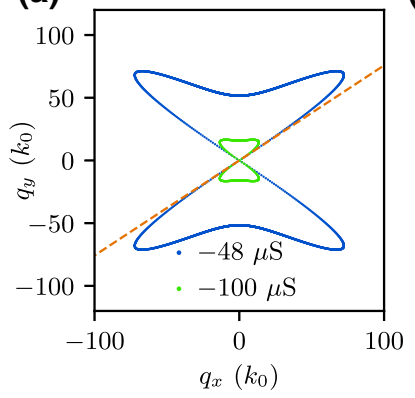

(b)

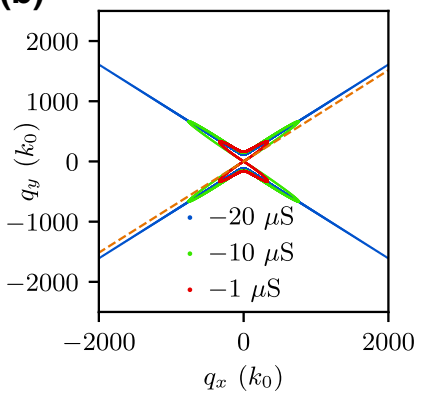

FIG. 6. Isofrequency contours for hyperbolic materials with different values of gain. $\sigma_{x x}=12-i 19 \mu \mathrm{S}, \sigma_{y y}=\operatorname{Re}\left(\sigma_{y y}\right)+$ $i 33 \mu \mathrm{S}) . \operatorname{Re}\left(\sigma_{y y}\right)$ is indicated in the legend in (a),(b).

Bilayer black phosphorus as a hyperbolic material also offers the possibility of spontaneous emission-rate enhancement through the Purcell effect $[46,47]$. We defer these results to the Appendix.

\section{CONCLUSION}

In conclusion, we show in this work how black phosphorus can be made hyperbolic across a broad spectral range, in particular in the visible wavelengths. We show how the spectral location of these hyperbolic regions could be tuned via electrostatic bias and strain. We further study the influence of optical gain on the hyperbolic plasmons and show the appearance of a new hyperbolic region in the infrared. The ease of such a tunability of the plasmons as shown in this work, opens up new opportunities for actively tunable flatland nanophotonics [48-50], particularly with regard to optoelectronic devices [51] in the direction of sensing wavefront control [52], nanoscale heat transfer [29], and spontaneous emission-rate enhancement [28].

\section{ACKNOWLEDGMENTS}

R.R. acknowledges financial support from the Spanish MINECO through Ramón y Cajal program, Grants No. RYC-2016-20663 and No. FIS2014-58445-JIN. M.I.K. acknowledges financial support from the European Research Council Advanced Grant Program (Contract No. 338957). S.Y. acknowledges financial support from National Key R \& D Program of China (Grant No. 2018FYA0305800) and National Science Foundation of China (Grant No. 11774269). A.K. acknowledges financial support from Science \& Engineering Research Board via Grant No. SB/S2/RJN-110/2017 and DST Grant No. DST/NM/NS/2018/49. Numerical calculations presented in this paper are performed on a supercomputing system in the Supercomputing Center of Wuhan University.

\section{APPENDIX: PURCELL FACTORS}

When an excited emitter is placed near a nanostructure supporting a photonic mode, the rate at which the former gets rid of its energy is modified compared to the case when the emitter is in free space. This phenomenon is referred to as Purcell enhancement or spontaneous-emission enhancement. Hyperbolic metasurfaces also offer the possibility of spontaneous-emissionrate enhancement through the Purcell effect [46,47]. Since bilayer black phosphorus supports hyperbolic plasmon modes, we present the possibility of Purcell enhancement in this system. In order to study spontaneous-emission-rate engineering in an anisotropic 2D material, we employ the formalism of Ref. [32], where

$$
\mathcal{S}=\frac{P}{P_{0}}=1+\frac{6 \pi}{k_{0}} \hat{\mathbf{n}} \cdot \mathfrak{I}\left\{G_{S}\left(\mathbf{r}_{\mathbf{0}}, \mathbf{r}_{\mathbf{0}} ; \omega\right)\right\} \cdot \hat{\mathbf{n}} .
$$

If we consider only the vertically polarized dipole emitter (i.e., polarized out of the plane of the black phosphorus sheet) placed a distance $d$ above the sheet, the scattered Green tensor simplifies to

$$
\begin{aligned}
\hat{\mathbf{n}} & \cdot\left\{\overline{\bar{G}}_{s}\left(\mathbf{r}_{\mathbf{0}}, \mathbf{r}_{\mathbf{0}} ; \omega\right)\right\} \cdot \hat{\mathbf{n}} \\
& =\frac{l}{8 \pi^{2}} \iint \Gamma_{\mathrm{pp}} \hat{\mathbf{n}} \cdot \overline{\bar{M}}_{\mathrm{pp}} \cdot \hat{\mathbf{n}} e^{2 l k_{z} d} d k_{x} d k_{y}
\end{aligned}
$$

(where pp denotes "p-polarized to p-polarized tensor element"). Here the two terms in the integrand are given by

$$
\hat{\mathbf{n}} \cdot \overline{\bar{M}} \cdot \hat{\mathbf{n}}=\frac{k_{\rho}^{2}}{k_{z} k_{0}^{2}}
$$

and

$$
\Gamma=\frac{-Z_{0} \sigma_{x x}^{\prime}\left(2 Z^{s}+Z_{0} \sigma_{y y}^{\prime}\right)+Z_{0}^{2} \sigma_{x y}^{\prime} \sigma_{y x}^{\prime}}{\left(2 Z^{s}+Z_{0} \sigma_{y y}^{\prime}\right)\left(2 Z^{p}+Z_{0} \sigma_{x x}^{\prime}\right)-Z_{0}^{2} \sigma_{x y}^{\prime} \sigma_{y x}^{\prime}},
$$

with $Z_{0}=\sqrt{\mu_{0} / \epsilon_{0}}, \quad Z^{s}=k_{z} / k_{0}, \quad Z^{p}=k_{0} / k_{z}, \quad$ and $\sigma^{\prime}$ denotes the rotated-surface conductivity tensor. If the material has no intrinsic off-diagonal contribution to the surface conductivity, then the components of the rotatedsurface conductivity tensor simplify to

$$
\begin{aligned}
& \sigma_{x x}^{\prime}=\frac{k_{x}^{2} \sigma_{x x}+k_{y}^{2} \sigma_{y y}}{k_{\rho}^{2}}, \\
& \sigma_{y y}^{\prime}=\frac{k_{x}^{2} \sigma_{y y}+k_{y}^{2} \sigma_{x x}}{k_{\rho}^{2}}, \\
& \sigma_{x y}^{\prime}=\sigma_{y x}^{\prime}=\frac{k_{x} k_{y}\left(\sigma_{y y}-\sigma_{x x}\right)}{k_{\rho}^{2}} .
\end{aligned}
$$

The result of this calculation is presented in Fig. 7, where we assume a $z$-polarized emitter located at a distance of 
(a)
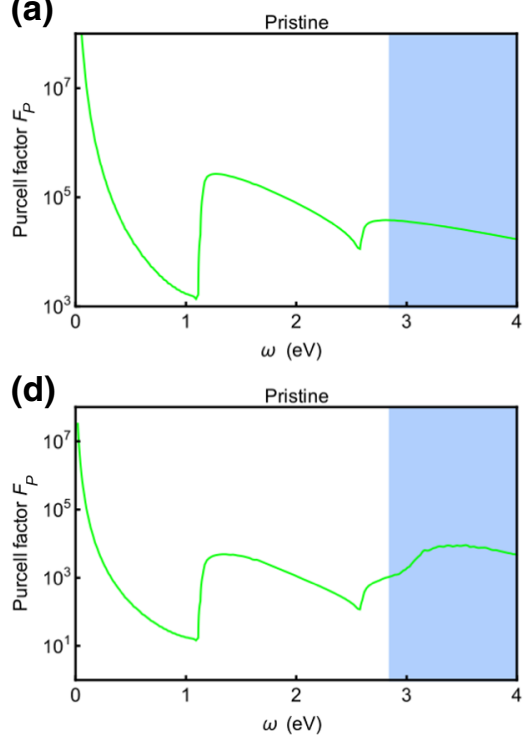

(b)

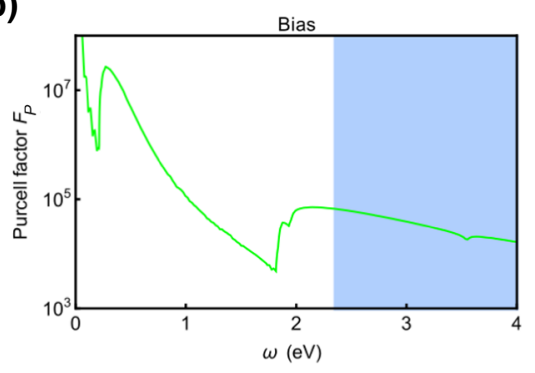

(e)

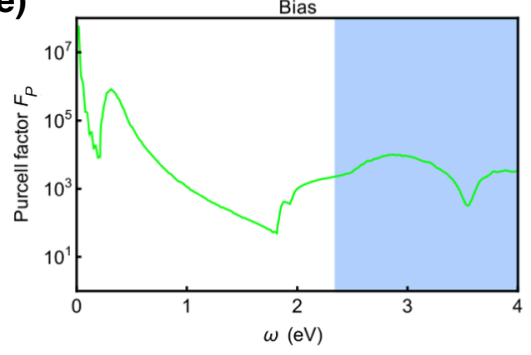

(c)

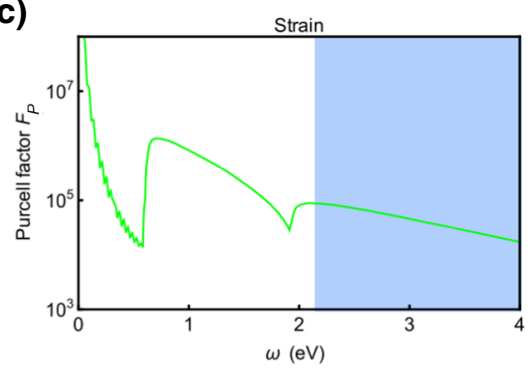

(f)

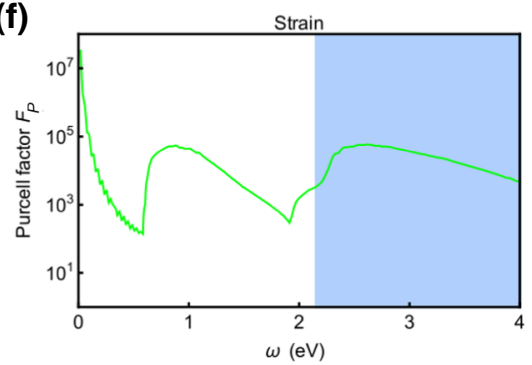

FIG. 7. (a)-(c) Purcell enhancement for a $z$-polarized emitter placed $1 \mathrm{~nm}$ above the $2 \mathrm{D}$ sheet without gain. Elliptical to hyperbolic transition is not observed in the Purcell spectra due to the high interband losses. (d)-(f):= In the low-loss regime (loss reduced by 100), one can identify this transition.

$1 \mathrm{~nm}$ from free-standing bilayer black phosphorus. Due to the large loss in the optical conductivity in the hyperbolic regions, a clear transition from the elliptical to hyperbolic regime [47] is not observed, as shown in Figs. 7(a)-7(c). The fact that this is due to the large losses is confirmed by carrying out similar calculations where the real part of the optical conductivity is reduced by a multiplicative factor of 100. In this case, one can clearly observe the elliptical to hyperbolic transition as shown in Figs. 7(d)-7(f). Moreover, the transition point of the Purcell factor is clearly shown to be tunable as a function of bias and strain.

[1] 2D Materials: Properties and Devices, edited by $\mathrm{P}$. Avouris, T. F. Heinz, and T. Low (Cambridge University Press, Cambridge, UK, 2017).

[2] R. Roldán, L. Chirolli, E. Prada, J. A. Silva-Guillén, P. SanJose, and F. Guinea, Theory of $2 \mathrm{~d}$ crystals: Graphene and beyond, Chem. Soc. Rev. 46, 4387 (2017).

[3] L. Li, Y. Yu, G. J. Ye, Q. Ge, X. Ou, H. Wu, D. Feng, X. H. Chen, and Y. Zhang, Black phosphorus field-effect transistors, Nat. Nano 9, 372 (2014).

[4] H. Liu, A. T. Neal, Z. Zhu, Z. Luo, X. Xu, D. Tománek, and P. D. Ye, Phosphorene: An unexplored 2d semiconductor with a high hole mobility, ACS Nano 8, 4033 (2014).

[5] A. Castellanos-Gomez, L. Vicarelli, E. Prada, J. O. Island, K. L. Narasimha-Acharya, S. I. Blanter, D. J. Groenendijk, M. Buscema, G. A. Steele, J. V. Alvarez, H. W. Zandbergen, J. J. Palacios, and H. S. J. van der Zant, Isolation and characterization of few-layer black phosphorus, 2D Mater. 1, 025001 (2014).

[6] F. Xia, H. Wang, and Y. Jia, Rediscovering black phosphorus as an anisotropic layered material for optoelectronics and electronics, Nat. Commun. 5, 4458 (2014).

[7] R. Roldán and A. Castellanos-Gomez, Black phosphorus: A new bandgap tuning knob, Nat. Photonics 11, 407 (2017).

[8] C. Lin, R. Grassi, T. Low, and A. S. Helmy, Multilayer black phosphorus as a versatile mid-infrared electro-optic material, Nano Lett. 16, 1683 (2016).

[9] R. Peng, K. Khaliji, N. Youngblood, R. Grassi, T. Low, and M. Li, Midinfrared electro-optic modulation in few-layer black phosphorus, Nano Lett. 17, 6315 (2017).

[10] W. S. Whitney, M. C. Sherrott, D. Jariwala, W.-H. Lin, H. A. Bechtel, G. R. Rossman, and H. A. Atwater, Field effect optoelectronic modulation of quantum-confined carriers in black phosphorus, Nano Lett. 17, 78 (2016).

[11] B. Deng, V. Tran, Y. Xie, H. Jiang, C. Li, Q. Guo, X. Wang, H. Tian, S. J. Koester, H. Wang, J. J. Cha, Q. Xia, L. Yang, and F. Xia, Efficient electrical control of thin-film black phosphorus bandgap, Nat. Commun. 8, 14474 (2017).

[12] Y. Liu, Z. Qiu, A. Carvalho, Y. Bao, H. Xu, S. J. Tan, W. Liu, A. Castro Neto, K. P. Loh, and J. Lu, Gate-tunable giant Stark effect in few-layer black phosphorus, Nano Lett. 17, 1970 (2017).

[13] J. Kim, S. S. Baik, S. H. Ryu, Y. Sohn, S. Park, B.-G. Park, J. Denlinger, Y. Yi, H. J. Choi, and K. S. Kim, Observation of tunable band gap and anisotropic dirac semimetal state in black phosphorus, Science 349, 723 (2015).

[14] J. Yang, R. Xu, J. Pei, Y. W. Myint, F. Wang, Z. Wang, S. Zhang, Z. Yu, and Y. Lu, Optical tuning of exciton and trion emissions in monolayer phosphorene, Light Sci. Appl. 4, e312 (2015).

[15] J. Quereda, P. San-Jose, V. Parente, L. Vaquero-Garzon, A. J. Molina-Mendoza, N. Agraït, G. Rubio-Bollinger, F. Guinea, R. Roldán, and A. Castellanos-Gomez, Strong modulation of optical properties in black phosphorus 
through strain-engineered rippling, Nano Lett. 16, 2931 (2016).

[16] A. S. Rodin, A. Carvalho, and A. H. Castro Neto, StrainInduced Gap Modification in Black Phosphorus, Phys. Rev. Lett. 112, 176801 (2014).

[17] Z. J. Xiang, G. J. Ye, C. Shang, B. Lei, N. Z. Wang, K. S. Yang, D. Y. Liu, F. B. Meng, X. G. Luo, L. J. Zou, Z. Sun, Y. Zhang, and X. H. Chen, Pressure-Induced Electronic Transition in Black Phosphorus, Phys. Rev. Lett. 115, 186403 (2015).

[18] T. Low, A. Chaves, J. Caldwell, A. Kumar, N. Fang, P. Avouris, T. Heinz, F. Guinea, L. Martin-Moreno, and F. Koppens, Polaritons in layered two-dimensional materials, Nat. Mater. 16, 182 (2017).

[19] R. W. Keyes, The electrical properties of black phosphorus, Phys. Rev. 92, 580 (1953).

[20] A. Poddubny, I. Iorsh, P. Belov, and Y. Kivshar, Hyperbolic metamaterials, Nat. Photonics 7, 948 (2013).

[21] O. Yermakov, A. Ovcharenko, M. Song, A. Bogdanov, I. Iorsh, and Y. S. Kivshar, Hybrid waves localized at hyperbolic metasurfaces, Phys. Rev. B 91, 235423 (2015).

[22] J. S. Gomez-Diaz, M. Tymchenko, and A. Alu, Hyperbolic Plasmons and Topological Transitions over Uniaxial Metasurfaces, Phys. Rev. Lett. 114, 233901 (2015).

[23] A. Nemilentsau, T. Low, and G. Hanson, Anisotropic 2d Materials for Tunable Hyperbolic Plasmonics, Phys. Rev. Lett. 116, 066804 (2016).

[24] Y. Yermakov, A. A. Hurshkainen, D. A. Dobrykh, P. V. Kapitanova, I. V. Iorsh, S. B. Glybovski, and A. A. Bogdanov, Experimental observation of hybrid TE-TM polarized surface waves supported by a hyperbolic metasurface, Phys. Rev. B 98, 195404 (2018).

[25] W. Ma, P. Alonso-González, S. Li, A. Y. Nikitin, J. Yuan, J. Martín-Sánchez, J. Taboada-Gutiérrez, I. Amenabar, P. Li, S. Vélez, Christopher Tollan, Zhigao Dai, Yupeng Zhang, Sharath Sriram, Kourosh Kalantar-Zadeh, ShuitTong Lee, Rainer Hillenbrand, and Qiaoliang Bao, In-plane anisotropic and ultra-low-loss polaritons in a natural van der Waals crystal, Nature 562, 557 (2018).

[26] Z. Zheng, N. Xu, S. L. Oscurato, M. Tamagnone, F. Sun, Y. Jiang, Y. Ke, J. Chen, W. Huang, W. L. Wilson, Shaozhi Deng, and Huanjun Chen, A mid-infrared biaxial hyperbolic van der waals crystal, arXiv:1809.03432 (2018).

[27] D. Correas-Serrano, J. Gomez-Diaz, A. A. Melcon, and A. Alù, Black phosphorus plasmonics: Anisotropic elliptical propagation and nonlocality-induced canalization, J. Opt. 18, 104006 (2016).

[28] H. N. S. Krishnamoorthy, Z. Jacob, E. Narimanov, I. Kretzschmar, and V. M. Menon, Topological transitions in metamaterials, Science 336, 205 (2012), https:// science.sciencemag.org/content/336/6078/205.full.pdf.

[29] S.-A. Biehs, M. Tschikin, and P. Ben-Abdallah, Hyperbolic Metamaterials as an Analog of a Blackbody in the Near Field, Phys. Rev. Lett. 109, 104301 (2012).

[30] C. T. Riley, J. S. T. Smalley, J. R. J. Brodie, Y. Fainman, D. J. Sirbuly, and Z. Liu, Near-perfect broadband absorption from hyperbolic metamaterial nanoparticles, Proc. Natl. Acad. Sci. 114, 1264 (2017).
[31] K. V. Sreekanth, Y. Alapan, M. ElKabbash, E. Ilker, M. Hinczewski, U. A. Gurkan, A. De Luca, and G. Strangi, Extreme sensitivity biosensing platform based on hyperbolic metamaterials, Nat. Mater. 15, 621 EP (2016).

[32] J. S. Gomez-Diaz, M. Tymchenko, and A. Alù, Hyperbolic metasurfaces: surface plasmons, light-matter interactions, and physical implementation using graphene strips [invited], Opt. Mater. Express 5, 2313 (2015).

[33] J. Shang, C. Cong, Z. Wang, N. Peimyoo, L. Wu, C. Zou, Y. Chen, X. Y. Chin, J. Wang, C. Soci, Wei Huang, and Ting $\mathrm{Yu}$, Room-temperature $2 \mathrm{~d}$ semiconductor activated vertical-cavity surface-emitting lasers, Nat. Commun. 8, 543 (2017).

[34] S. Wu, S. Buckley, J. R. Schaibley, L. Feng, J. Yan, D. G. Mandrus, F. Hatami, W. Yao, J. Vučković, A. Majumdar, and Xiaodong $\mathrm{Xu}$, Monolayer semiconductor nanocavity lasers with ultralow thresholds, Nature 520, 69 (2015).

[35] A. N. Rudenko, and M. I. Katsnelson, Quasiparticle band structure and tight-binding model for single- and bilayer black phosphorus, Phys. Rev. B 89, 201408 (2014).

[36] A. Rudenko, S. Yuan, and M. Katsnelson, Toward a realistic description of multilayer black phosphorus: From $G W$ approximation to large-scale tight-binding simulations, Phys. Rev. B 92, 085419 (2015).

[37] H. Suzuura and T. Ando, Phonons and electron-phonon scattering in carbon nanotubes, Phys. Rev. B 65, 235412 (2002).

[38] P. San-Jose, V. Parente, F. Guinea, R. Roldán, and E. Prada, Inverse Funnel Effect of Excitons in Strained Black Phosphorus, Phys. Rev. X 6, 031046 (2016).

[39] Q. Wei and X. Peng, Superior mechanical flexibility of phosphorene and few-layer black phosphorus, Appl. Phys. Lett. 104, 251915 (2014).

[40] T. Low, P.-Y. Chen, and D. Basov, Superluminal plasmons with resonant gain in population inverted bilayer graphene, Phys. Rev. B 98, 041403 (2018).

[41] A. F. Page, F. Ballout, O. Hess, and J. M. Hamm, Nonequilibrium plasmons with gain in graphene, Phys. Rev. B 91, 075404 (2015).

[42] T. Low, A. S. Rodin, A. Carvalho, Y. Jiang, H. Wang, F. Xia, and A. H. Castro Neto, Tunable optical properties of multilayer black phosphorus thin films, Phys. Rev. B 90, 075434 (2014).

[43] G. Zhang, S. Huang, A. Chaves, C. Song, V. O. Özçelik, T. Low, and H. Yan, Infrared fingerprints of few-layer black phosphorus, Nat. Commun. 8, 14071 (2017).

[44] G. Ni, L. Wang, M. Goldflam, M. Wagner, Z. Fei, A. McLeod, M. Liu, F. Keilmann, B. Özyilmaz, A. C. Neto, J. Hone, M. M. Fogler, and D. N. Basov, Ultrafast optical switching of infrared plasmon polaritons in high-mobility graphene, Nat. Photonics 10, 244 (2016).

[45] C. H. Lui, K. F. Mak, J. Shan, and T. F. Heinz et al., Ultrafast Photoluminescence from Graphene, Phys. Rev. Lett. 105, 127404 (2010).

[46] E. M. Purcell, Spontaneous emission probabilities at radio frequencies, Phys. Rev. 69, 681 (1946).

[47] M. N. Gjerding, R. Petersen, T. G. Pedersen, N. A. Mortensen, and K. S. Thygesen, Layered van der Waals 
crystals with hyperbolic light dispersion, Nat. Commun. 8, 320 (2017).

[48] I. Liberal, Y. Li, and N. Engheta, Reconfigurable epsilonnear-zero metasurfaces via photonic doping, Nanophotonics 7, 1117 (2018).

[49] L. Lu, R. E. Simpson, and S. K. Valiyaveedu, Active hyperbolic metamaterials: Progress, materials and design, J. Opt. 20, 103001 (2018).
[50] J. S. T. Smalley, F. Vallini, X. Zhang, and Y. Fainman, Dynamically tunable and active hyperbolic metamaterials, Adv. Opt. Photon. 10, 354 (2018).

[51] Metasurfaces - from science to applications, Nanophotonics 7, 949 (2018).

[52] S. M. Kamali, E. Arbabi, A. Arbabi, and A. Faraon, A review of dielectric optical metasurfaces for wavefront control, Nanophotonics 7, 1041 (2018). 